\title{
Adherence to and acceptability of the Mediterranean diet amongst young adults in the North East of England
}

\author{
L. Francis, J. Young and J. Lara \\ Department of Applied Sciences, Faculty of Health and Life Sciences, University of Northumbria at Newcastle, \\ NE1 8ST, UK.
}

Adherence to the Mediterranean diet (MD) has been shown to confer significant health benefits including, but not limited to, increased longevity and reduced incidence of cardiovascular disease ${ }^{(1)}$. Despite this, evidence on the adherence to and acceptability of the MD guidelines in non-Mediterranean countries is still scarce.

This study aimed to investigate current adherence to and acceptability of the MD amongst young British adults. The Bristol Online Survey tool was used to create an anonymous survey to investigate barriers to healthy eating and MD adherence using a previously developed score ${ }^{(1)}$. Data collected was analysed using independent t-tests, Chi-square tests and partial correlation analysis controlling for covariates.

554 individuals ( $86.9 \%$ female) responded to this survey. Participants' mean age was 28.2 years SD 8.31, and mean BMI was 25.67 $\mathrm{kg} / \mathrm{m}^{2}$ SD 11.47. Overall, 92.9\% were Caucasian and $91.7 \%$ lived in the North East of England. Mean MD adherence score, out of a maximum of 14 points, was 5.24 SD 2.09, with no difference between men (mean 5.31 SD 1.92) and women (mean 5.23 SD 2.11); $\mathrm{p}=0.780$. Partial correlation analysis controlling for sex and BMI, showed that age was positively associated with MD adherence score $(r=0.184, p<0 \cdot 001)$, while BMI was negatively associated with MD score $(r=-0 \cdot 100, p=0 \cdot 019)$. In addition, MD adherence score was negatively associated with the number of barriers to healthy eating reported $(r=-0.439, p<0.001)$ while controlling for age, sex and BMI.

The MD score components with the lowest prevalence were "consuming more than four tablespoons of olive oil per day" (3.8 \% of participants) and "drinking seven or more glasses of wine per week" (4.5\%). Conversely, $84.0 \%$ preferentially consumed more white than red meat, and $87.8 \%$ used a tomato-based cooking sauce two or more times per week. Statistically significant differences between men and women were only seen in the consumption of red meat, with $8.3 \%$ of men eating less than one portion of red meat per week, compared to $24.6 \%$ of women; $p=0.002 .43 \%$ of volunteers reported that they 'know a little bit about the MD' and an additional $10.5 \%$ stated that they 'fully understand the concept of the MD'. Most participants (45.4\%) selected Mediterranean as their favourite cuisine, followed by Asian (27.4\%) and British or American (14.5\%) cuisines.

Acceptability of the MD was high in this population, with $65.7 \%$ of participants finding MD guidelines acceptable; acceptability of individual MD guidelines ranged from $90.9 \%$ for consumption of "more than three portions of vegetables per day", to $20.9 \%$ for "more than four tablespoons of olive oil per day". Partial correlation analysis controlling for age, sex and BMI showed that MD acceptability was positively associated with MD adherence score $(r=0.226, p<0.001)$. In addition MD acceptability was positively associate with age $(r=0.219, \mathrm{p}<0.001)$, independently of sex and BMI. Finally, MD acceptability was negatively associated with the number of barriers to healthy eating $(\mathrm{r}=-0 \cdot 150, \mathrm{p}<0 \cdot 001)$.

In conclusion, this study suggests that adherence to the overall MD is currently low, however the MD guidelines are seen as acceptable by this population including overweight and obese young individuals. These results should be of value for those interested in developing interventions to promote healthy eating among young adults in the UK.

1. Estruch R, Ros E, Salas-Salvadó J et al. (2013) N Engl J 14, 1279-90. 